\section{Revisão sistemática dos estudos epidemiológicos sobre discriminação interpessoal e saúde mental}

\author{
Systematic review of epidemiological studies on \\ interpersonal discrimination and mental health
}

\author{
Revisión sistemática de estudios epidemiológicos \\ sobre la discriminación interpersonal y salud \\ mental
}

Janaina Brugnera Goto 1

Paulo Francisco Mastella Couto 1

João Luiz Bastos 1

\section{Resumo}

The article describes epidemiological studies on the relationship between interpersonal discrimination and mental health outcomes, updating previous literature reviews on the topic. The review included 34 papers published in PubMed from 2000 to 2010, 68\% of which were based on convenience samples and $82 \%$ with a cross-sectional design. Positive and statistically significant associations were observed between discrimination and adverse mental health outcomes, particularly substance use, depression, and alcoholrelated disorders. Only one third of the studies explicitly adopted a specific theoretical framework to interpret the examined relationships. Mirroring previous reviews, discrimination was positively and consistently associated with adverse mental health outcomes. However, future studies should employ robust designs for causal inference, use discrimination instruments with good psychometric properties, and adopt theoretical frameworks to interpret their findings.

Prejudice; Mental Health; Causality; Epidemiologic Studies
Foram caracterizados estudos epidemiológicos que avaliaram a relação entre discriminação interpessoal e condições de saúde mental, atualizando revisões prévias sobre o tema. Identificaram-se 34 artigos publicados entre 2000 e 2010 no PubMed, dos quais 68\% utilizaram amostras de conveniência e 82\% o delineamento transversal. Observaram-se associações positivas e estatisticamente significativas entre discriminação e condições adversas de saúde mental, especialmente uso de substâncias, depressão e transtornos associados ao uso de álcool. Somente um terço dos estudos explicitou um referencial teórico para interpretar as relações examinadas. Similarmente às revisões anteriores, pode-se afirmar que as experiências discriminatórias se associam positiva e consistentemente com desfechos adversos de saúde mental. Entretanto, investigações futuras deverão empregar delineamentos mais robustos para a inferência causal, utilizar instrumentos de discriminação com boas propriedades psicométricas e adotar referencial teórico específico para interpretar os resultados produzidos.

Preconceito; Saúde Mental; Causalidade; Estudos Epidemiológicos 


\section{Introdução}

A discriminação tem impacto considerável sobre os grupos contra os quais é praticada, implica a negação de seus direitos e afeta sua individualidade 1. Conceitualmente, a discriminação pode ser entendida como um "tratamento diferencial e frequentemente desigual a pessoas, formal ou informalmente agrupadas em uma categoria particular. [...] Apesar de ser amiúde uma ação individual, é também um padrão social de comportamento agregado" 2 (p. 1182). Ações discriminatórias envolvem preferências culturais e são enraizadas em atitudes cotidianas, geralmente apoiadas por tradições de longa duração ${ }^{3}$. Algumas formas de discriminação têm sido descritas na literatura. Particularmente, a discriminação interpessoal, foco desta revisão, é entendida como um ato isolado, realizado por um indivíduo com base em seus próprios preconceitos 4 .

A investigação com enfoque na discriminação como um fator determinante de condições e comportamentos em saúde tem ocorrido com base em estudos empíricos, publicados principalmente a partir da década de 1980 5. As revisões de literatura sobre o assunto têm demonstrado associação das experiências discriminatórias com condições e comportamentos adversos, como hipertensão; aterosclerose 6; condições adversas de saúde mental 6,7,8,9,10,11,12,13; falta ao trabalho por motivo de doença 8; tabagismo e alcoolismo 6,11 ; uso de outras drogas 11 ; e problemas de conduta ${ }^{11}$. Relato materno de discriminação também tem sido associado a desfechos negativos da gestação, como prematuridade, baixo e muito baixo peso ao nascer de seus filhos 11 .

Têm sido descritos três mecanismos por meio dos quais as experiências discriminatórias poderiam afetar a saúde 12,13. Todos consideram as experiências discriminatórias como formas de estresse relativamente imprevisíveis e de caráter tanto agudo quanto crônico. Em primeiro lugar, a discriminação afetaria a saúde de forma direta, provocando distúrbios psicológicos e agravos mentais, como a ansiedade e sintomas depressivos. Segundo, adotando-se comportamentos deletérios à saúde, por exemplo, tabagismo ou abandono de comportamentos saudáveis, como boa alimentação, atividade física e aderência a tratamentos medicamentosos. Finalmente, por intermédio das respostas fisiológicas do organismo ao estresse, incluindo alterações relacionadas aos aparelhos cardiovascular e gastrointestinal, sistema neuroendócrino, e função imunológica, sensibilidade à dor e dor crônica 12,13 .

Os resultados mais consistentes observados nesses estudos são aqueles para desfechos de saúde mental 6,7,8,9,10,11,12,13. Williams et al. 6, numa revisão de 32 estudos originais, observaram que $62 \%(n=26)$ daqueles que avaliaram a relação entre discriminação e distresse psicológico demonstraram associação positiva entre estas variáveis, e, dos 22 estudos que avaliaram outros desfechos de saúde mental, 18 (82\%) também relataram associações na mesma direção. Por sua vez, Paradies 9 observou que, em $72 \%$ das 148 vezes em que desfechos adversos de saúde mental foram avaliados nos trabalhos revisados, notou-se uma relação positiva e estatisticamente significante destas condições com experiências discriminatórias.

Uma revisão de Pachter \& Coll 11 sobre racismo - incluindo discriminação - e saúde infantil identificou 26 estudos que o associaram a problemas de saúde mental/comportamental, tais como depressão e uso de substâncias como tabaco, álcool e drogas ilícitas. Keyes 10, em trabalho eminentemente teórico, apresentou resultados de estudos com amostra representativa dos Estados Unidos (Midlife in the United States - MIDUS) que demonstraram associação entre discriminação e desfechos de saúde mental. Um deles apontou forte associação da discriminação com níveis reduzidos de bem-estar psicológico; outro, com risco e prevalência aumentados de desfechos adversos de saúde mental.

Pascoe \& Smart-Richman 12, por sua vez, observaram, além da associação entre discriminação e pior estado de saúde mental, que esta relação parece ser igualmente forte para todos os tipos de desfechos de saúde mental analisados. De 107 estudos que avaliaram um total de 500 associações da discriminação com a saúde mental, 448 (90\%) demonstraram que níveis mais altos de discriminação percebida estiveram associados a estados de saúde mental negativos, sendo que $69 \%(n=345)$ destas associações foram estatisticamente significantes.

Apenas uma revisão de literatura 7 avaliou exclusivamente desfechos de saúde mental e sua relação com experiências discriminatórias. Neste trabalho, foram analisados 15 estudos, sendo que, em todos eles, a discriminação atuou como fator deletério para as condições de saúde mental avaliadas. Os desfechos estudados incluíram, por exemplo, distresse psicológico, sintomas depressivos, satisfação com a vida e felicidade. Essa revisão apresentou informações de cada estudo original acerca do país de origem, o ano de publicação, a caracterização etnicorracial dos participantes, o tamanho da amostra, a faixa etária dos entrevistados e o instrumento utilizado para aferir discriminação. No entanto, não foram explicitamente informados: a forma de seleção ou tipo da amostra (conveniência ou probabilística), 
o tipo de discriminação avaliada (interpessoal ou institucional; racial, de gênero, idade, classe etc.) e os referenciais teóricos utilizados pelos autores para interpretar as relações de interesse.

Tendo em vista essas limitações e o período de tempo decorrido desde a publicação da primeira revisão sobre o assunto, o objetivo do presente trabalho foi atualizar a revisão da literatura mencionada 7 e caracterizar os estudos epidemiológicos que avaliaram a relação entre discriminação e condições de saúde mental quanto ao tipo de discriminação mensurada e os referenciais teóricos acionados para interpretar as relações de interesse. Espera-se, com esta revisão, descrever o estado atual das pesquisas epidemiológicas sobre discriminação e desfechos de saúde mental, identificando fragilidades e lacunas neste conhecimento, as quais deverão ser enfrentadas por pesquisas futuras na área. Em particular, busca-se responder à seguinte pergunta de pesquisa: qual a direção, consistência e significância estatística das associações encontradas entre discriminação e desfechos de saúde mental, investigadas nos estudos originais sobre o tema?

\section{Métodos}

\section{Estratégia de busca e fonte bibliográfica}

O presente trabalho seguiu as recomendações do documento PRISMA (Preferred Reporting Items for Systematic Reviews and Meta-Analyses) 14, o qual visa a orientar a divulgação de revisões sistemáticas da literatura e meta-análises na área da saúde. Foi conduzida uma pesquisa eletrônica exclusivamente na base PubMed, para localizar estudos epidemiológicos que avaliaram a relação entre discriminação e condições adversas de saúde mental, com ênfase em transtornos de humor, ansiedade e associados ao uso de substâncias, publicados de 2000 a 2010. A busca foi realizada sem a imposição de outros limites, como sexo, idade dos participantes ou idioma da publicação. Para a construção da chave de busca, foi consultado o tesauro da base bibliográfica, de modo a encontrar os termos controlados (MeSH - Medical Subject Headings) que melhor identificassem os trabalhos a serem incluídos na revisão. Foi realizada consulta à estrutura hierárquica do tesauro, optando-se pelos termos mais amplos, isto é, aqueles pertencentes aos níveis mais altos das árvores analisadas. Também foi incluído um termo livre para aumentar a sensibilidade da chave de busca, que segue: (prejudice[mesh] or discrimination [tiab]) and (anxiety disorders [mesh] or mood disorders[mesh] or substance-related disorders[mesh]). Referências bibliográficas de artigos selecionados não foram checadas, tampouco foram consultados especialistas no assunto para localizar artigos não publicados.

\section{Identificação dos estudos e critérios de inclusão}

Esta chave resultou em 1.118 publicações, em busca realizada no dia 29 de junho de 2011. Os títulos e, se necessário, os resumos, e na ausência destes, o texto completo, foram analisados pela autora J.B.G. quanto à relevância para inclusão na revisão. Incluíram-se estudos epidemiológicos que apresentassem uma medida de discriminação interpessoal autorrelatada como principal exposição ou mediador, e que avaliassem condições de saúde mental como desfechos. Os trabalhos que enfocaram a prevalência de condições de saúde mental em diferentes grupos etnicorraciais, sem avaliar as experiências de discriminação, foram excluídos. Excluíram-se também estudos que não se propuseram a aferir a discriminação de modo isolado das demais exposições pesquisadas, considerando-a como um construto separado. Essa exclusão se estendeu àqueles trabalhos que abordaram, como exposições, conceitos relacionados como preconceito, estigma e desvalorização de grupo racial. Também foram excluídos os estudos que avaliaram a discriminação direcionada a portadores de condições psiquiátricas.

\section{Extração de dados}

Foram examinados, então, os textos completos dos artigos selecionados. A primeira autora procedeu à extração de dados com um formulário especificamente desenvolvido para atender aos objetivos da revisão, testado previamente numa amostra de cinco artigos, em conjunto com os demais autores. Divergências na extração de dados foram discutidas até que se obtivesse consenso. Extraíram-se informações acerca do ano de publicação, país e instituição de origem, periódico, tamanho e forma de seleção da amostra, sexo, faixa etária, caracterização etnicorracial e orientação sexual dos participantes, tipo de discriminação mensurada, utilização ou não de referencial teórico, argumentação do referencial (quando utilizado), dados sobre os instrumentos empregados no estudo, incluindo nome, número de itens, submissão a algum processo de adaptação; e sobre as associações encontradas, sua direção, significância estatística e restrição a algum grupo populacional, fatores mediadores e moderadores das associações, caso presentes. Por referencial teórico entende-se um modelo explicativo que se utiliza de conceitos e relações para a proposição e interpretação de as- 
sociações entre fenômenos. Fator mediador pode ser compreendido como uma terceira variável ou constructo que intervém entre dois constructos relacionados - exposição e desfecho -, produzindo uma relação indireta ou mediada. Representa o mecanismo por intermédio do qual a variável independente é capaz de influenciar a variável dependente. Por fator moderador compreende-se um terceiro constructo que modifica a direção ou a magnitude da relação entre duas variáveis.

A direção de associação foi classificada em positiva (relações diretamente proporcionais), negativa (associações inversamente proporcionais), e sem associação (ocorrência do desfecho não varia de acordo com as categorias da exposição à discriminação). A direção ou forma de associação foi descrita para todos os cruzamentos realizados, e não somente para aqueles identificados como estatisticamente significantes. Isso permitiu estimar a proporção de associações estatisticamente significantes entre todas aquelas avaliadas pelos estudos originais. Sempre que possível foram consideradas as associações que foram ajustadas para fatores de confusão em modelos de regressão multivariáveis ou outras estratégias de análise estatística

\section{Análises estatísticas}

Os dados produzidos foram digitados, uma única vez, com o auxílio do programa EpiData, versão 3.1 (Epidata Association, Odense, Dinamarca), com controles automáticos de consistência e amplitude (a planilha resultante desta digitação está disponível, mediante consulta aos autores). Analisou-se os dados com o pacote estatístico Stata, versão 11.1 (Stata Corp., College Station, Estados Unidos), por meio de frequências absolutas e relativas. A direção e a significância estatística das associações testadas foram apresentadas em uma tabela de contingência.

\section{Resultados}

Dos 1.118 trabalhos inicialmente identificados na fonte bibliográfica, 1.080 publicações foram excluídas por não atenderem aos critérios de inclusão, por meio da leitura de seus títulos e/ou resumos. Em seguida, excluíram-se mais quatro artigos, após a leitura de seus conteúdos na íntegra, por não se tratarem de estudos originais ou não apresentarem medida de discriminação interpessoal autorrelatada como a principal exposição. Esse processo resultou em 34 estudos $15,16,17,18,19,20,21,22,23,24,25,26,27,28,29,30,31,32,33,34,35,36$, $37,38,39,40,41,42,43,44,45,46,47,48$ a serem analisados na presente revisão (Figura 1).
A maioria foi publicada entre 2008 e 2010 (56\%), nos Estados Unidos (94\%), conforme apresentado na Tabela 1. Os periódicos com maior número de estudos publicados foram Journal of Youth \& Adolescence, American Journal of Public Health e Journal of Health \& Social Behavior, todos com três publicações, cada. Quanto ao tipo de trabalho, a maioria (82\%) utilizou desenho transversal, e os outros seis (20\%) foram longitudinais. Grande parte dos estudos utilizou amostras selecionadas por conveniência $(68 \%)$, com 1.001 a 34.653 indivíduos $(n=17)$. Adolescentes e adultos representaram a faixa etária estudada em $44 \%$ dos trabalhos $(n=12)$, apenas adolescentes $11 \%(n=3)$ e crianças, adolescentes e adultos $11 \%$ $(\mathrm{n}=3)$. Na maioria dos estudos (88\%), indivíduos de ambos os sexos foram pesquisados, com exceção de três, que investigaram somente participantes do sexo feminino, e um, exclusivamente sujeitos do sexo masculino. Cerca de $50 \%$ dos estudos $(n=16)$ abrangeram múltiplos grupos etnicorraciais, $21 \%$ estudaram exclusivamente negros $(n=7)$ e $12 \%$ exclusivamente latinos $(n=4)$. Três artigos classificaram os participantes quanto à orientação sexual, destes, dois incluíram participantes heterossexuais. As demais categorias utilizadas para a classificação referiram-se aos comportamentos que diferem da heterossexualidade - dois trabalhos utilizaram categorias diferentes para participantes bissexuais com preferência por homossexualidade, e bissexuais com preferência por heterossexualidade (Tabela 2).

Conforme a Tabela 3, as escalas mais utilizadas foram Experiences of Discrimination, em seis artigos, e Schedule of Racist Events, em três. Pouco mais de um quarto dos estudos não utilizou escala previamente elaborada para medir a exposição de interesse $(n=9)$. Dois estudos serviramse simultaneamente de dois instrumentos para avaliar as experiências discriminatórias, enquanto os demais, apenas um. Em 74\% dos trabalhos, o tipo de discriminação primariamente avaliado foi a etnicorracial $(n=25)$, seguida da discriminação por orientação sexual, em $12 \%$ dos estudos $(\mathrm{n}=4)$, e de gênero, em $6 \%$ dos trabalhos $(\mathrm{n}=2)$. A discriminação por múltiplas motivações foi examinada em dois estudos. Um único artigo não explicitou essa informação. O período/modo de exposição à discriminação mais avaliado pelos estudos foi "ao longo da vida" em nove deles, seguido de "crônica diária" e "no último ano" em quatro estudos cada.

Conforme apresentado na Tabela 4 , foram analisadas 66 relações entre a exposição e os desfechos, das quais 50 (76\%) foram positivas, sem restrição a qualquer dos grupos de indivíduos investigados. Dentre as demais $(n=16)$, dez 
Figura 1

Fluxograma da revisão de literatura.

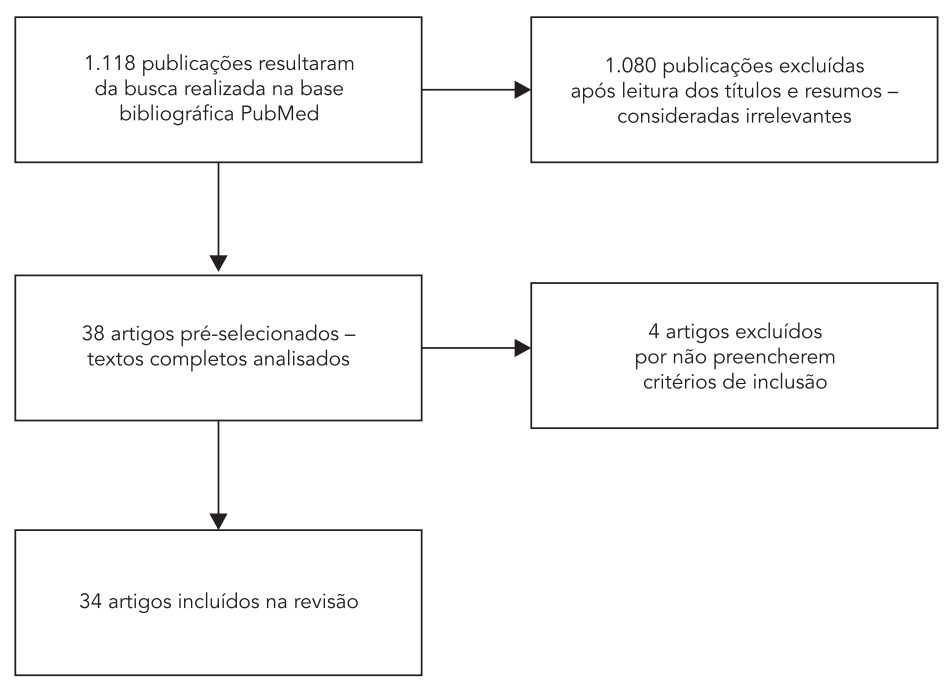

Tabela 1

Características bibliográficas dos trabalhos incluídos na revisão de literatura. Base bibliográfica PubMed, 2011.

\begin{tabular}{llc}
\hline Características & $\mathbf{n}$ & $\%$ \\
\hline Ano de publicação & & 5,9 \\
$2000-2001$ & 2 & - \\
$2002-2003$ & - & 14,7 \\
$2004-2005$ & 5 & 23,5 \\
$2006-2007$ & 8 & 23,5 \\
$2008-2009$ & 8 & 32,4 \\
2010 & 11 & 38,2 \\
Autores por publicação & & 52,9 \\
$\leq 3$ & 13 & 8,8 \\
$4-6$ & 18 & 8,8 \\
$\geq 7$ & 3 & 8,8 \\
Periódico de publicação & & 8,8 \\
Journal of Youth \& Adolescence & 3 & 5,9 \\
American Journal of Public Health & 3 & 5,9 \\
Journal of Health \& Social Behavior & 3 & 5,9 \\
Journal of Counseling Psychology & 2 & 55,9 \\
Journal of Personality \& Social Psychology & 2 & 2,9 \\
Women's Health Issues & 2 & 94,1 \\
Demais periódicos com um artigo cada & 19 & 2,9 \\
País de origem do estudo & & \\
Estados Unidos & 32 & \\
Holanda & 1 & \\
México & 1 & \\
Total & 34 & \\
\hline
\end{tabular}


Tabela 2

Características metodológicas dos estudos incluídos na revisão de literatura. Base bibliográfica PubMed, 2011.

\begin{tabular}{|c|c|c|}
\hline Característica & $\mathrm{n}$ & $\%$ \\
\hline \multicolumn{3}{|l|}{ Tipo de estudo } \\
\hline Transversal & 28 & 82,4 \\
\hline Coorte & 6 & 17,6 \\
\hline \multicolumn{3}{|l|}{ Forma de seleção dos participantes } \\
\hline Conveniência & 23 & 67,6 \\
\hline Amostragem estratificada & 6 & 17,6 \\
\hline Amostragem complexa & 4 & 11,8 \\
\hline Não descrita & 1 & 2,9 \\
\hline \multicolumn{3}{|l|}{ Tamanho da amostra } \\
\hline$\leq 100$ & 1 & 2,9 \\
\hline $101-200$ & 3 & 8,8 \\
\hline $201-1.000$ & 13 & 38,2 \\
\hline $1.001-34.653$ & 17 & 50,0 \\
\hline \multicolumn{3}{|l|}{ Sexo dos participantes } \\
\hline Feminino & 3 & 8,8 \\
\hline Masculino & 1 & 2,9 \\
\hline Ambos & 30 & 88,2 \\
\hline \multicolumn{3}{|l|}{ Faixa etária dos participantes (anos) * } \\
\hline Crianças (6-12) & 1 & 3,7 \\
\hline Crianças e adolescentes (6-18) & 2 & 7,4 \\
\hline Crianças, adolescentes, adultos e idosos (6+) & 2 & 7,4 \\
\hline Crianças, adolescentes e adultos (6-65) & 3 & 11,1 \\
\hline Adolescentes (13-18) & 3 & 11,1 \\
\hline Adolescentes e adultos (13-64) & 12 & 44,4 \\
\hline Adolescentes, adultos e idosos (13+) & 2 & 7,4 \\
\hline Adultos (19-64) & 1 & 3,7 \\
\hline Adultos e idosos (19+) & 1 & 3,7 \\
\hline \multicolumn{3}{|l|}{ Classificação etnicorracial dos participantes ** } \\
\hline Negros & 7 & 21,2 \\
\hline Latinos & 4 & 12,1 \\
\hline Indígenas & 2 & 6,1 \\
\hline Mexicanos & 2 & 6,1 \\
\hline Filipinos & 1 & 3,0 \\
\hline Somalianos & 1 & 3,0 \\
\hline Múltiplos grupos etnicorraciais *** & 16 & 48,5 \\
\hline \multicolumn{3}{|l|}{ Orientação sexual dos participantes \# } \\
\hline $\begin{array}{l}\text { Heterossexual, principalmente heterossexual, bissexual, principalmente homossexual, } \\
\text { homossexual, gay ou lésbica }\end{array}$ & 1 & 33,3 \\
\hline Bissexual/homossexual, bissexual/heterossexual e homossexual & 1 & 33,3 \\
\hline Heterossexual, gay ou lésbica, bissexual e transgênero & 1 & 33,4 \\
\hline Total & 34 & 100,0 \\
\hline
\end{tabular}

* Informação não disponível para sete estudos;

** Informação não disponível para um estudo;

*** Esta categoria inclui trabalhos com (a) brancos, negros, hispânicos, asiáticos, originários do oriente médio e outros, (b) hispânicos, negros nascidos nos Estados Unidos e originários do sudeste asiático, (c) filipinos, indígenas originários da Ásia, vietnamitas, coreanos, japoneses e multirraciais, (d) brancos, negros, asiáticos, (e) negros, brancos, latinos e asiáticos, (f) negros, chineses, hispânicos e brancos, (g) do Marrocos, de outros países não-ocidentais, das Antilhas Holandesas, do Suriname, da Turquia e de países ocidentais, (h) chineses, filipinos, vietnamitas, de outros países asiáticos e multirraciais, (i) brancos, hispânicos, negros e outros, (j) negros, latinos/mexicanos e brancos, (I) brancos, negros, indígenas americanos, asiáticos/originários das Ilhas do Pacífico e hispânicos, (m) brancos e negros, (n) indígenas americanos, originários do sudeste asiático, negros nascidos nos Estados Unidos, negros nascidos na África, hispânicos e brancos;

\# Informação não disponível para 31 estudos. 
Métodos e instrumentos para aferição das experiências discriminatórias nos trabalhos incluídos na revisão de literatura. Base bibliográfica PubMed, 2011.

\begin{tabular}{|c|c|c|}
\hline Métodos e instrumentos & n & $\%$ \\
\hline \multicolumn{3}{|l|}{ Trabalhos que utilizaram, pelo menos, um instrumento para avaliar experiências } \\
\hline \multicolumn{3}{|l|}{ discriminatórias - informações sobre o instrumento } \\
\hline \multicolumn{3}{|l|}{ Escala para aferição da discriminação } \\
\hline Experiences of Discrimination & 6 & 17,6 \\
\hline Schedule of Racist Events & 3 & 8,8 \\
\hline Everyday Discrimination Scale & 2 & 5,9 \\
\hline Schedule of Sexist Events & 2 & 5,9 \\
\hline Racism and Life Experiences Scale & 1 & 2,9 \\
\hline Outras escalas & 11 & 32,4 \\
\hline Não foi utilizada escala & 9 & 26,5 \\
\hline \multicolumn{3}{|l|}{ Tipo de discriminação avaliada } \\
\hline Etnicorracial & 25 & 73,5 \\
\hline Orientação sexual & 4 & 11,8 \\
\hline Gênero & 2 & 5,9 \\
\hline $\begin{array}{l}\text { Etnicorracial, orientação sexual e gênero, tempo de encarceramento, idade, } \\
\text { uso de drogas e pobreza }\end{array}$ & 2 & 5,9 \\
\hline Não informada & 1 & 2,9 \\
\hline \multicolumn{3}{|l|}{ Exposição cumulativa à discriminação } \\
\hline Ao longo da vida & 8 & 23,5 \\
\hline Crônica diária & 4 & 11,8 \\
\hline No último ano & 4 & 11,8 \\
\hline Nos últimos três meses & 1 & 2,9 \\
\hline Outras exposições & 7 & 20,6 \\
\hline Não informada & 10 & 29,4 \\
\hline Total & 34 & 100,0 \\
\hline \multicolumn{3}{|l|}{$\begin{array}{l}\text { Trabalhos que utilizaram dois instrumentos para avaliar experiências discriminatórias, } \\
\text { simultaneamente - informações sobre o segundo instrumento }\end{array}$} \\
\hline \multicolumn{3}{|l|}{ Escala para aferição da discriminação } \\
\hline Everyday Discrimination Scale & 1 & 33,3 \\
\hline Escala de racismo & 1 & 33,3 \\
\hline Unfair Events Scale & 1 & 33,4 \\
\hline \multicolumn{3}{|l|}{ Tipo de discriminação avaliada } \\
\hline Etnicorracial & 2 & 66,7 \\
\hline $\begin{array}{l}\text { Etnicorracial, orientação sexual e gênero, tempo de encarceramento, idade, } \\
\text { uso de drogas e pobreza }\end{array}$ & 1 & 33,3 \\
\hline \multicolumn{3}{|l|}{ Exposição cumulativa à discriminação } \\
\hline Ao longo da vida & 1 & 33,3 \\
\hline Durante a juventude, na vida adulta & 1 & 33,3 \\
\hline Não informada & 1 & 33,4 \\
\hline Total & 3 & 100,0 \\
\hline \multicolumn{3}{|l|}{$\begin{array}{l}\text { Trabalhos que utilizaram três instrumentos para avaliar experiências discriminatórias, } \\
\text { simultaneamente - informações sobre o terceiro instrumento }\end{array}$} \\
\hline \multicolumn{3}{|l|}{ Escala para aferição da discriminação } \\
\hline Escala de racismo & 1 & 100,0 \\
\hline \multicolumn{3}{|l|}{ Tipo de discriminação avaliada } \\
\hline Etnicorracial & 1 & 100,0 \\
\hline \multicolumn{3}{|l|}{ Exposição cumulativa à discriminação } \\
\hline Durante a juventude, na vida adulta & 1 & 100,0 \\
\hline Total & 1 & 100,0 \\
\hline
\end{tabular}


Resultados da associação entre experiências discriminatórias e desfechos de saúde mental nos trabalhos incluídos na revisão de literatura. Base bibliográfica PubMed, 2011.

\begin{tabular}{|c|c|c|c|c|c|c|c|}
\hline \multirow[t]{2}{*}{ Desfecho } & \multicolumn{2}{|c|}{ Associação global * } & \multicolumn{3}{|c|}{ Associação condicional ** } & \multirow{2}{*}{$\begin{array}{c}\text { Sem } \\
\text { associação }\end{array}$} & \multirow[t]{2}{*}{ Tota } \\
\hline & $\begin{array}{l}\text { Positiva } \\
(\% \star \star \star *)\end{array}$ & $\begin{array}{c}\text { Negativa } \\
(\% * \star *)\end{array}$ & $\begin{array}{l}\text { Positiva } \\
(\% \star \star \star *)\end{array}$ & $\begin{array}{c}\text { Negativa } \\
(\% \star \star \star *)\end{array}$ & $\begin{array}{c}\text { Estratos populacionais em que } \\
\text { foi observada associação } \\
\text { condicional ** }\end{array}$ & & \\
\hline Ansiedade \# & $4(75,0)$ & $-(-)$ & $1(100,0)$ & $-(-)$ & Mulheres & - & 5 \\
\hline Uso de substâncias \#\# & $17(94,1)$ & $1(100,0)$ & $1(100,0)$ & $-(-)$ & Brancos & 2 & 21 \\
\hline $\begin{array}{l}\text { Transtornos associados ao uso } \\
\text { de álcool \#\# }\end{array}$ & $7(85,7)$ & $-(-)$ & $4(100,0)$ & $-(-)$ & $\begin{array}{c}\text { Hispânicos; negros nascidos na } \\
\text { África; negros }\end{array}$ & 2 & 13 \\
\hline $\begin{array}{l}\text { Depressão e transtornos } \\
\text { associados } \S\end{array}$ & $11(100,0)$ & $-(-)$ & $2(100,0)$ & $-(-)$ & Homens & - & 13 \\
\hline $\begin{array}{l}\text { Sintomas de estresse pós- } \\
\text { traumático }\end{array}$ & $4(100,0)$ & $-(-)$ & $-(-)$ & $-(-)$ & - & - & 4 \\
\hline Transtornos psicóticos & $1(100,0)$ & $-(-)$ & $-(-)$ & $-(-)$ & - & - & 1 \\
\hline Saúde mental geral & $-(-)$ & $1(100,0)$ & $-(-)$ & $-(-)$ & - & - & 1 \\
\hline Tabagismo §§ & $5(100,0)$ & $-(-)$ & $2(100,0)$ & $-(-)$ & $\begin{array}{l}\text { Originários do Sudeste Asiático; } \\
\text { negros; brancos }\end{array}$ & - & 7 \\
\hline Transtornos mentais comuns & $1(100,0)$ & $-(-)$ & $-(-)$ & $-(-)$ & - & - & 1 \\
\hline Total & $50(96,0)$ & $2(100,0)$ & $10(100,0)$ & $-(-)$ & - & 4 & 66 \\
\hline
\end{tabular}

* Associação global consiste naquela observada em todo o conjunto dos indivíduos investigados, não restrita a estratos específicos;

** Associação condicional corresponde à associação verificada apenas em grupos específicos da população em estudo;

*** Proporção das associações que foram estatisticamente significativas (valor de probabilidade $<0,05$ );

\# Inclui sintomas de ansiedade, problemas de internalização (problema de ansiedade, problema de ansiedade/depressão);

\#\# Inclui consumo de álcool, tabaco e drogas ilícitas, uso de drogas ilícitas, uso de maconha, uso de cocaína, uso de crack, uso de anfetamina, uso de heroína, uso de substâncias controladas, uso precoce de maconha;

\#\#\# Inclui dependência de álcool, consumo excessivo de álcool (binge drinking e heavy drinking), consumo de álcool por número de dias nos últimos 30 dias,

consumo de álcool no último ano, abuso de álcool nos últimos 12 meses;

§ Inclui sintomas depressivos, automutilação, ideação suicida, tentativa de suicídio;

$\S \S$ Inclui número de cigarros fumados por dia.

associações foram positivas, mas condicionais a determinados estratos populacionais, quatro não apresentaram associação, e duas evidenciaram associação negativa. Os desfechos avaliados em relação às experiências discriminatórias foram categorizados pelos autores desta revisão em nove grupos. Entre os desfechos mais estudados estão o uso de substâncias, com 21 (32\%) do total de associações testadas; a depressão, e os transtornos associados ao uso de álcool, cada um com 13 (20\%). Diferentemente dos demais, os desfechos referentes à ansiedade, uso de substâncias e uso de álcool apresentaram, cada um, uma associação positiva estatisticamente não significante, correspondendo, respectivamente, a $25 \%, 6 \%$ e $14 \%$ do total de associações testadas. As associações positivas condicionais ocorreram em homens, mulheres, brancos, negros/afroamericanos, hispânicos, e originários do sudeste asiático. Dentre as 66 associações, um total de
15 foram provenientes de estudos longitudinais. Dessas, nove foram positivas globais, duas foram positivas condicionais e quatro não apresentaram qualquer direção. Onze das associações testadas avaliaram desfechos referentes ao uso de substâncias. De modo geral, as associações estudadas indicaram que o relato mais frequente de experiências discriminatórias esteve associado com maior ocorrência dos desfechos adversos avaliados.

Pouco mais de um terço (34\%) dos estudos utilizou referencial teórico específico para interpretar as relações examinadas. Um dos referenciais teóricos utilizados por parte dos autores trata de estratégias de enfrentamento, que explicariam, por exemplo, maiores taxas de uso de substâncias entre indivíduos que relatam mais experiências discriminatórias. Entre outras, as estratégias de enfrentamento incluíram avaliação menos negativa da experiência discriminatória, e 
ruminação - referindo-se à lembrança repetitiva de determinado evento. Modelos teóricos específicos, que também acionam a temática do "enfrentamento" são o Modelo Integrativo, proposto por Garcia-Coll et al. 28 , e o Modelo de Estresse e Enfrentamento, de Folkman \& Lazarus 42 . Outros referenciais teóricos utilizados foram o Minority Stress Model, de Meyer 37, Cognitive Models of Depression, de Abramson et al. 35, The Stress Model of Prejudice and Discrimination, de Clark et al. $31 \mathrm{e}$ o Modelo de Hurh \& Kim 43, que afirma que, para minorias étnicas, a marginalidade e as barreiras institucionais tornam-se especialmente nocivas para a saúde mental do indivíduo, em períodos iniciais de adaptação e após longo período de residência nos Estados Unidos.

Os trabalhos que analisaram desfechos relacionados ao trauma citam, como referenciais teóricos, o Modelo de Psicopatologia do Desenvolvimento do Trauma 25, e Estresse Traumático Baseado em Raça, de Carter 49. Esse último modelo teoriza que grande parte do trauma associado à discriminação étnica e/ou racial estaria relacionado a uma percepção do evento como emocionalmente doloroso, negativo, repentino e fora do controle do indivíduo, critérios estes que são consistentes com a definição de trauma de Carlson 27,40. Também foram citados como referenciais o Fenômeno de Auto-culpa 39 e o Rejection-Identification Model 33.

Dentre os estudos que adotaram algum referencial teórico, dez avaliaram potenciais mediadores e/ou moderadores dos efeitos das experiências discriminatórias em relação aos desfechos examinados. Foi relatada moderação sobre o uso de substâncias por aculturação linguística e tempo de residência nos Estados Unidos 34, presença de pais apoiadores 30 , e autoestima elevada 17 . Um estudo 42 relatou que raça/etnia moderou a relação entre a discriminação de gênero, estresse e uso de drogas durante a vida, exclusivamente para o subgrupo de mulheres latinas. Mediadores relatados incluíram raiva 30,44 , comportamento classificado como "deliquente" ${ }^{44}$, e hostilidade 30 . O estudo de Almeida et al. 16, diferentemente dos demais trabalhos, avaliou a discriminação percebida como fator mediador entre o status de ser gay/lésbica/bissexual/transgênero e três desfechos associados à depressão. Esse encontrou mediação positiva para os três desfechos, porém, com maior significância estatística para jovens do sexo masculino. Em outras palavras, a discriminação consistiu em fator que liga o status de ser gay/lésbica/ bissexual/transgênero e os desfechos mencionados, especialmente em indivíduos jovens do sexo masculino.

\section{Discussão}

Uma maior quantidade absoluta de artigos publicados nos últimos anos analisados evidencia um interesse crescente dos pesquisadores pelo assunto. O periódico com o maior número de publicações, Journal of Youth \& Adolescence, é direcionado a um público multidisciplinar, interessado no tema da juventude e da adolescência, publicando preferencialmente artigos que valorizam as implicações políticas decorrentes dos estudos. Em segundo lugar, no que se refere ao número absoluto de publicações, o American Journal of Public Health é a revista oficial da Associação Americana de Saúde Pública (APHA), e tem como um de seus objetivos publicar as melhores pesquisas científicas no campo da saúde pública; e o Journal of Health \& Social Behavior, um periódico de sociologia médica, que veicula artigos que aplicam conceitos e métodos sociológicos para a compreensão da saúde e temas relacionados. A política editorial desse último periódico privilegia estudos que fomentam a compreensão teórica dos processos pelos quais os fatores sociais e de saúde humana estão inter-relacionados.

A condução da maioria dos estudos nos Estados Unidos revela uma concentração de trabalhos sobre esse tema no país, bem como a necessidade de que investigações semelhantes sejam conduzidas em outros contextos de investigação. Essas seriam importantes para checar se os resultados encontrados naquele país são também consistentes em outros contextos socioculturais. Porém, considerando que a quase totalidade dos questionários para aferir discriminação publicados na literatura tem origem nos Estados Unidos e enfatiza, com poucas exceções, a discriminação de tipo racial 50, avaliar este fenômeno, por exemplo, no Brasil com tais instrumentos, implica enfrentar desafios de ordem teórica e metodológica importantes. Em primeiro lugar, cabe considerar que o conceito de raça é interpretado de maneira distinta, se compararmos ambos os países em questão. Os brasileiros empregam com mais frequência o termo cor, no lugar de raça, quando da realização de classificações raciais 51 . O uso de termos distintos reflete que, no Brasil, são especialmente - mas não unicamente - características fenotípicas, que fundamentam a classificação das pessoas quanto à cor (ou raça). Isso está em certo contraste com o que ocorre nos Estados Unidos, onde a ideia de origem ou ancestralidade predomina, norteando o pertencimento de pessoas a grupos socialmente definidos 52 .

Outra diferença importante, ainda no que se refere à classificação de cor e, por consequência, à discriminação, está na existência de múltiplas categorias de classificação. Nos Estados Unidos 
predomina o modelo binário, que não prevê gradações expressivas entre o preto e o branco 52 . Por sua vez, no Brasil, o contínuo ou gradiente de cores é característico e está baseado na atribuição de distinções sociais a diversas tonalidades e terminologias utilizadas para localizar, de um modo bastante particular, os indivíduos num espectro de cor, que se estende do preto ao branco ${ }^{52}$. Há que se considerar, inclusive, o fenômeno do branqueamento na classificação de cor brasileira 53. Existe no país uma tendência de negros e mulatos em ascensão econômica de serem aceitos socialmente como brancos. Esses aspectos estão intimamente relacionados com a discriminação: tendo em vista que a cor depende de outros marcadores de posição social, especialmente o status socioeconômico; indivíduos no extremo escuro do contínuo de cores podem não ser tratados de modo discriminatório por apresentarem alto poder aquisitivo.

Cabe igualmente ressaltar as características distintivas da discriminação racial no Brasil, mantendo o contraponto com os Estados Unidos. O conceito chave para descrever a discriminação racial seria o da miscigenação, mestiçagem, mistura ou democracia racial no Brasil. Já nos Estados Unidos o termo equivalente seria o da segregação 54. Enquanto a discriminação racial no Brasil se manifestaria de maneira imbricada com a ideia de miscigenação/democracia racial, a segregação, entendida como a separação física e social entre negros e brancos, seria a principal forma de expressão da discriminação nos Estados Unidos 54 . Sintetizando, a ideia de cor (e não necessariamente de raça, significando origem ou ancestralidade), a existência de múltiplas categorias de classificação, sua relação com posição socioeconômica e, por fim, as peculiaridades da discriminação no Brasil, indicam que os instrumentos estadunidenses dificilmente abarcariam estas especificidades, não sendo adequados sem que haja adaptações pertinentes à mensuração das experiências discriminatórias em nosso contexto. Outra possibilidade à iniciativa de adaptar instrumentos concebidos em outros contextos socioculturais seria utilizar aqueles já desenvolvidos no Brasil, como tem sido recentemente observado na literatura 55 .

Os resultados de associação dos estudos de coorte incluídos nesta revisão indicam não apenas que experiências discriminatórias estão associadas a desfechos negativos de saúde mental, mas sugerem com maior poder de inferência que a exposição pode estar entre os fatores causais das condições e comportamentos adversos de saúde analisados. Quanto ao desenho transversal da maioria dos estudos, deve-se salientar uma limitação importante deste tipo de delineamento. Considerando que, nos estudos transversais, tanto a exposição quanto os desfechos são aferidos em um único momento no tempo, torna-se impossível avaliar se a exposição foi temporalmente anterior ou posterior ao desfecho investigado. Isso limita o estabelecimento de relações temporais (e causais) entre os fatores, por exemplo, a experiência discriminatória e o uso de substâncias. Com relação ao tipo de amostragem, apesar de amostras representativas terem sido utilizadas em dez casos, a maior parte selecionou seus participantes por conveniência, impossibilitando que os resultados encontrados sejam generalizados para populações-alvo previamente identificadas.

O tamanho das amostras, expressivo em parcela significativa dos estudos, demonstra o esforço dos pesquisadores em produzir trabalhos com resultados mais precisos. O estudo de indivíduos pertencentes a amplas faixas etárias permite uma análise dos efeitos da discriminação nas diferentes fases do ciclo vital. Por outro lado, a utilização de um mesmo instrumento de aferição de experiências discriminatórias, aplicado simultaneamente a indivíduos de faixas etárias diferentes pode implicar uma avaliação menos rigorosa das experiências discriminatórias, tendo em vista que estas tendem a ser marcadamente distintas ao longo da vida.

Os estudos com múltiplos grupos etnicorraciais, equivalendo à metade dos trabalhos revisados, permitem identificar diferentes resultados para cada grupo populacional. Apesar de reconhecer que motivações distintas podem gerar experiências discriminatórias diferentes, a literatura sugere a utilização de amostras com diversidade etnicorracial 11. Note-se que, para isso, é fundamental que os instrumentos utilizados para aferição das experiências discriminatórias tenham sido elaborados com esse objetivo. Estudos com apenas um grupo etnicorracial avaliaram principalmente populações negras ou latinas, demonstrando que ainda há um interesse maior dos pesquisadores por esses grupos populacionais em relação aos demais. Quanto à classificação dos participantes por orientação sexual, apesar de ter sido realizada em apenas três trabalhos, não foi uniforme. Assim como para a classificação por grupo etnicorracial, cada estudo utilizou uma categorização própria, não consistente entre eles.

De modo geral, a ênfase na aferição da discriminação racial e na abordagem de populações negras nos estudos revisados reflete uma tendência já observada por outros autores nos trabalhos sobre preconceito no campo das ciências humanas e sociais, especialmente no âmbito da psicologia e da sociologia 56: pesquisadores 
dedicados ao escrutínio científico deste constructo frequentemente lidam com a questão da raça e assumem que o preconceito e o racismo se tratam de conceitos intimamente relacionados. Além disso, os modelos conceituais que procuram explicar o preconceito, seus antecedentes e suas consequências destacam o papel dos perpetradores e dos comportamentos discriminatórios, restritos ao nível interpessoal. Tendo em vista que a literatura sobre discriminação e saúde mental se origina, em grande parte, dos Estados Unidos, seria razoável esperar que a produção científica sobre as relações de interesse para esta revisão procedesse deste país, com predomínio da questão racial, avaliada em nível individual. De certa forma, isso reflete quanto o racismo foi (e ainda é) um aspecto central na constituição dos Estados Unidos como nação, em que a escravidão foi tomada como um elemento fundamental na manutenção da riqueza do país, e a discriminação, considerada até mesmo necessária, sendo apoiada por ideologias de cunho racista que a justificavam 56 . A proeminência da questão racial nos estudos sobre preconceito e discriminação está em contraste com o que se observa nas investigações cujo fenômeno central é o estigma. Nesse caso, valorizam-se análises dos estigmas associados às doenças, incapacidades ou "desvios da normalidade" 56. Pesquisas futuras abordando a discriminação e suas interfaces com condições e comportamentos em saúde deverão, idealmente, ampliar o foco sobre os tipos de discriminação avaliados, incluindo também outros eixos de desigualdades, como gênero, orientação sexual, classe, dentre outros.

A escala mais utilizada nos estudos revisados, Experiences of Discrimination (EOD), é um instrumento curto, com nove itens, baseado em outro previamente aplicado no estudo Coronary Artery Risk Development in Young Adults (CARDIA) 57. Foi desenvolvido e testado quanto à validade e confiabilidade por Krieger et al. 58 . A pergunta inicial feita ao participante questiona se ele "já sofreu discriminação, foi impedido de fazer algo, foi perturbado ou feito sentir-se inferior", em alguma das nove situações listadas, por sua raça, etnia ou cor. As situações são: na escola; procurando emprego; no trabalho; procurando moradia; buscando atendimento médico; recebendo serviços em um restaurante ou loja; solicitando crédito, empréstimo bancário ou hipoteca; na rua ou em um local público; da polícia ou em tribunal/de justiça. Para resposta positiva a cada situação, segue a pergunta sobre o número de vezes que isso ocorreu (com três opções de resposta: uma vez, duas ou três vezes, quatro ou mais vezes). O instrumento apresentou alta confiabilidade (avaliada pelo coeficiente alfa de Cronbach igual ou maior que 0,74 , e coeficiente teste-reteste igual a 0,70 ), e alta validade (constatada pela mais alta correlação com um constructo de discriminação basal, em comparação com outros instrumentos de aferição de experiências discriminatórias). Entretanto, Conde \& Gorman 59 criticam esse instrumento por ser demasiadamente genérico, e por considerarem que a pergunta inicial agrupa em um constructo único diversos tipos de experiências, que podem ter efeitos distintos sobre a saúde.

A segunda escala mais utilizada, Schedule of Racist Events (SRE) 60, é um questionário de autorrelato com 18 itens, que avalia a frequência de eventos discriminatórios racistas recentes (no último ano) e durante toda a vida do indivíduo, e mede o grau em que esta discriminação foi avaliada como estressante para o próprio respondente. Cada um desses três módulos forma uma subescala específica. Os cálculos para cada subescala demonstraram alta confiabilidade (por meio de coeficientes de consistência interna maiores que 0,93 e da técnica de metades partidas - split-half - com coeficientes maiores que 0,90 ).

Os resultados dos trabalhos revisados indicam que existe uma associação entre discriminação autorrelatada e condições negativas de saúde mental. A maior parte das relações examinadas confirma a expectativa dos pesquisadores, sendo estatisticamente significante. Isso se dá de modo diferente dos resultados de estudos com desfechos de saúde física como, por exemplo, pressão arterial, que não apresentam resultados tão consistentes ${ }^{61}$. A variedade de grupos que apresentou associações condicionais (por exemplo, homens, mulheres, brancos, negros) demonstra inexistência de um padrão claro. Essas associações condicionais isoladas, e em poucos estudos, indicam que a relação entre discriminação e condições adversas de saúde mental se aplica mais às populações em geral do que a subgrupos específicos.

Os trabalhos revisados aferiram discriminação autorrelatada, uma forma subjetiva de avaliação. Como observou Meyer 62, essa maneira de medir eventos discriminatórios, em contraste com formas objetivas de aferição, apresenta uma limitação importante - diversos fatores influenciam a percepção, ou seja, o modo como o indivíduo avalia o evento, e o relato destes eventos. Esse autor teoriza que indivíduos de grupos minoritários, que são motivados a detectar a discriminação por autoproteção, também são motivados a ignorá-la para preservar as relações sociais e a satisfação com a vida. Portanto, é possível inferir que indivíduos mais saudáveis utilizam estratégias que os levam a subestimar os eventos discriminatórios. Essa limitação de aferição 
da exposição é ainda mais preocupante quando examinada em conjunto com desfechos de saúde mental, graças a um possível viés de atenuação do impacto da discriminação percebida sobre a saúde. Além desse entrave, cabe mencionar que os instrumentos de discriminação utilizados nas pesquisas em saúde ora analisadas têm recebido outras críticas 63 . Em primeiro lugar, é preciso considerar que as experiências discriminatórias constituem fenômenos dotados de grande subjetividade e complexidade. A caracterização de uma experiência como discriminatória vai depender dos motivos atribuídos para sua ocorrência - assim o serão aquelas experiências motivadas por questões injustas como, por exemplo, a simples vinculação a grupos socialmente definidos. Claramente, os motivos atribuídos a um tratamento ou comportamento humano irão variar de acordo com a perspectiva de vítimas, perpetradores e eventuais testemunhas. Além desse aspecto fundamental, as experiências de discriminação relatadas pelos participantes dos estudos podem ter sido confusas, de modo que os mesmos podem não se sentir seguros para fazer afirmações sobre sua ocorrência. E, nos casos de ambiguidade de manifestação, pode haver tanto subestimação quanto superestimação da ocorrência das experiências discriminatórias 63 . Outra sorte de fatores que pode influenciar o relato de discriminação nos trabalhos analisados inclui a modalidade de produção de dados utilizada (entrevista face a face, aplicada com auxílio de computador, questionário autopreenchível etc.), a relação estabelecida entre entrevistador e entrevistado, o formato das perguntas e o contexto no qual a entrevista é conduzida 63 . Todos esses aspectos são relevantes e devem ser cuidadosamente considerados pelos pesquisadores interessados no assunto.
Reconhecemos como limitação deste trabalho que a consulta a uma única base de dados tem o potencial de ignorar outros artigos relevantes à temática. Entretanto, esta revisão foi mais ampla e metodologicamente rigorosa do que trabalhos prévios, publicados na literatura, abordando as mesmas relações 7 . Ademais, a PubMed tem sido a fonte bibliográfica de eleição de revisões sobre o assunto 9,12,50, na medida em que consiste na maior e mais conhecida fonte bibliográfica da área da saúde. Ainda, tendo em vista a predominância de estudos norte-americanos na investigação da relação entre discriminação e desfechos de saúde 13 , bem como a boa abrangência que a PubMed apresenta de trabalhos estadunidenses, compreende-se que a restrição a esta fonte bibliográfica provavelmente não alterou as conclusões produzidas. Como diferencial da revisão de literatura prévia 7 , expusemos os resultados de associação dos estudos revisados, facilitando sua visualização e compreensão. Trabalhos futuros deverão empregar desenhos longitudinais, sofisticar a classificação dos participantes quanto à orientação sexual, utilizar instrumentos de aferição previamente avaliados quanto às características psicométricas e fazer uso de referencial teórico específico para interpretar os resultados encontrados.

Esta revisão evidenciou que, apesar de diversos aspectos metodológicos e de embasamento teórico dos estudos epidemiológicos sobre esse assunto serem passíveis de aprimoramento, os resultados dos trabalhos publicados após a última revisão da literatura sobre discriminação e saúde mental 7 apontaram na mesma direção, e permitem afirmar que as experiências discriminatórias se associam positiva e consistentemente com desfechos adversos de saúde mental. 


\section{Resumen}

Fueron caracterizados los estudios epidemiológicos que evaluaron la relación entre la discriminación interpersonal y salud mental, actualizando revisiones previas sobre el tema. Se identificaron 34 artículos publicados entre 2000 y 2010 a través del PubMed, de los cuales el 68\% utilizaron muestras de conveniencia y el 82\% fueron estudios transversales. Se observaron asociaciones positivas y estadisticamente significativas entre la discriminación y condiciones adversas de salud mental, especialmente consumo de substancias, depresión y los trastornos asociados al consumo de alcohol. Sólo un tercio de los estudios explicitó un marco teórico para interpretar las relaciones examinadas. Al igual que en las revisiones anteriores, se puede afirmar que las experiencias discriminatorias se asocian positiva y consistentemente con resultados adversos de salud mental. Sin embargo, investigaciones futuras deberán emplear diseños más robustos para la inferencia causal, utilizar instrumentos de discriminación con buenas propiedades psicométricas y adoptar un marco teórico específico para interpretar los resultados producidos.

Prejuicio; Salud Mental; Causalidad; Estudios Epidemiológicos

\section{Colaboradores}

J. B. Goto contribuiu com a concepção e elaboração do estudo, incluindo a redação da primeira versão do texto. P. F. M. Couto participou da elaboração do estudo e da revisão crítica do texto. J. L. Bastos participou da concepção do estudo, análise dos dados, redação de trechos do texto e revisão crítica de sua versão final.

\section{Referências}

1. Pettigrew TF, Taylor MC. Discrimination. In: Borgatta EF, Montgomery RJV, editors. Encyclopedia of sociology. $2^{\text {nd }}$ Ed. New York: MacMillian Reference; 2000. p. 3481.

2. Law I. Discrimination. In: Ritzer G, editor. Blackwell encyclopedia of sociology. Oxford: Blackwell; 2007. p. 1182-4.

3. Lemert C. Discrimination. In: Turner BS, editor. Cambrigde dictionary of sociology. Cambrigde: Cambridge University Press; 2006. p. 708.

4. Mallick K. Individual discrimination. In: Magill FN, editor. International encyclopedia of sociology. London: Fitzroy Dearborn Publishers; 1995. p. 373-7.

5. Krieger N. Discrimination and health. In: Berkman LF, Kawachi I, editors. Social epidemiology. New York: Oxford University Press; 2000. p. 36-75.
6. Williams DR, Neighbors HW, Jackson JS. Racial/ ethnic discrimination and health: findings from community studies. Am J Public Health 2003; 93:200-8.

7. Williams DR, Williams-Morris R. Racism and mental health: the African American experience. Ethn Health 2000; 5:243-68.

8. McKenzie K. Tackling the root cause. There are clear links between racism and the higher rates of mental illness among ethnic minority groups. Ment Health Today 2004; 30-2.

9. Paradies Y. A systematic review of empirical research on self-reported racism and health. Int $\mathrm{J}$ Epidemiol 2006; 35:888-901.

10. Keyes CL. The Black-White paradox in health: flourishing in the face of social inequality and discrimination. J Pers 2009; 77:1677-706. 
11. Pachter LM, Coll CG. Racism and child health: a review of the literature and future directions. J Dev Behav Pediatr 2009; 30:255-63.

12. Pascoe EA, Smart Richman L. Perceived discrimination and health: a meta-analytic review. Psychol Bull 2009; 135:531-54.

13. Williams DR, Mohammed SA. Discrimination and racial disparities in health: evidence and needed research. J Behav Med 2009; 32:20-47.

14. Moher D, Liberati A, Tetzlaff J, Altman DG. Preferred reporting items for systematic reviews and meta-analyses: the PRISMA statement. PLoS Med 2009; 6:e1000097.

15. Veling W, Selten JP, Susser E, Laan W, Mackenbach JP, Hoek HW. Discrimination and the incidence of psychotic disorders among ethnic minorities in The Netherlands. Int J Epidemiol 2007; 36:761-8.

16. Almeida J, Johnson RM, Corliss HL, Molnar BE, Azrael D. Emotional distress among LGBT youth: the influence of perceived discrimination based on sexual orientation. J Youth Adolesc 2009; 38:1001-14.

17. Banks KH, Kohn-Wood LP, Spencer M. An examination of the African American experience of everyday discrimination and symptoms of psychological distress. Community Ment Health J 2006; 42:555-70.

18. Berg SH. Everyday sexism and posttraumatic stress disorder in women: a correlational study. Violence Against Women 2006; 12:970-88.

19. Borrell LN, Jacobs Jr. DR, Williams DR, Pletcher MJ, Houston TK, Kiefe CI. Self-reported racial discrimination and substance use in the Coronary Artery Risk Development in Adults Study. Am J Epidemiol 2007; 166:1068-79.

20. Borrell LN, Roux AV, Jacobs Jr. DR, Shea S, Jackson SA, Shrager S, et al. Perceived racial/ethnic discrimination, smoking and alcohol consumption in the Multi-Ethnic Study of Atherosclerosis (MESA). Prev Med 2010; 51:307-12.

21. Buchanan RL, Smokowski PR. Pathways from acculturation stress to substance use among latino adolescents. Subst Use Misuse 2009; 44:740-62.

22. Burgess D, Tran A, Lee R, van Ryn M. Effects of perceived discrimination on mental health and mental health services utilization among gay, lesbian, bisexual and transgender persons. J LGBT Health Res 2007; 3:1-14.

23. Canady RB, Bullen BL, Holzman C, Broman C, Tian Y. Discrimination and symptoms of depression in pregnancy among African American and White women. Womens Health Issues 2008; 18:292-300.

24. Chae DH, Takeuchi DT, Barbeau EM, Bennett GG, Lindsey JC, Stoddard AM, et al. Alcohol disorders among Asian Americans: associations with unfair treatment, racial/ethnic discrimination, and ethnic identification (the national Latino and Asian Americans study, 2002-2003). J Epidemiol Community Health 2008; 62:973-9.

25. Ellis BH, MacDonald HZ, Lincoln AK, Cabral HJ. Mental health of Somali adolescent refugees: the role of trauma, stress, and perceived discrimination. J Consult Clin Psychol 2008; 76:184-93.
26. Finch BK, Kolody B, Vega WA. Perceived discrimination and depression among Mexican-origin adults in California. J Health Soc Behav 2000; 41:295-313.

27. Flores E, Tschann JM, Dimas JM, Pasch LA, de Groat CL. Perceived racial/ethnic discrimination, posttraumatic stress symptoms, and health risk behaviors among Mexican American adolescents. J Couns Psychol 2010; 57:264-73.

28. Gaylord-Harden NK, Cunningham JA. The impact of racial discrimination and coping strategies on internalizing symptoms in African American youth. J Youth Adolesc 2009; 38:532-43.

29. Gee GC, Delva J, Takeuchi DT. Relationships between self-reported unfair treatment and prescription medication use, illicit drug use, and alcohol dependence among Filipino Americans. Am J Public Health 2007; 97:933-40.

30. Gibbons FX, Etcheverry PE, Stock ML, Gerrard M, Weng CY, Kiviniemi M, et al. Exploring the link between racial discrimination and substance use: what mediates? What buffers? J Pers Soc Psychol 2010; 99:785-801.

31. Gibbons FX, Gerrard M, Cleveland MJ, Wills TA, Brody G. Perceived discrimination and substance use in African American parents and their children: a panel study. J Pers Soc Psychol 2004; 86:517-29.

32. Gibbons FX, Yeh HC, Gerrard M, Cleveland MJ, Cutrona C, Simons RL, et al. Early experience with racial discrimination and conduct disorder as predictors of subsequent drug use: a critical period hypothesis. Drug Alcohol Depend 2007; 88 Suppl 1:S27-37.

33. Khaylis A, Waelde LC, Bruce EJ. The role of ethnic identity in the relationship of race-related stress to PTSD symptoms among young adults. J Trauma Dissociation 2007; 8:91-105.

34. Kulis S, Marsiglia FF, Nieri T. Perceived ethnic discrimination versus acculturation stress: influences on substance use among Latino youth in the Southwest. J Health Soc Behav 2009; 50:443-59.

35. Lambert SF, Herman KC, Bynum MS, Ialongo NS. Perceptions of racism and depressive symptoms in African American adolescents: the role of perceived academic and social control. J Youth Adolesc 2009; 38:519-31.

36. Les Whitbeck B, Chen X, Hoyt DR, Adams GW. Discrimination, historical loss and enculturation: culturally specific risk and resiliency factors for alcohol abuse among American Indians. J Stud Alcohol 2004; 65:409-18.

37. McCabe SE, Bostwick WB, Hughes TL, West BT, Boyd CJ. The relationship between discrimination and substance use disorders among lesbian, gay, and bisexual adults in the United States. Am J Public Health 2010; 100:1946-52.

38. Okamoto J, Ritt-Olson A, Soto D, Baezconde-Garbanati L, Unger JB. Perceived discrimination and substance use among Latino adolescents. Am J Health Behav 2009; 33:718-27. 
39. Ortiz-Hernandez L, Garcia Torres MI. Efectos de la violéncia y la discriminación en la salud mental de bisexuales, lesbianas y homosexuales de la Ciudad de México. Cad Saúde Pública 2005; 21:913-25.

40. Pieterse AL, Carter RT, Evans SA, Walter RA. An exploratory examination of the associations among racial and ethnic discrimination, racial climate, and trauma-related symptoms in a college student population. J Couns Psychol 2010; 57:255-63.

41. Potochnick SR, Perreira KM. Depression and anxiety among first-generation immigrant Latino youth: key correlates and implications for future research. J Nerv Ment Dis 2010; 198:470-7.

42. Ro A, Choi KH. Effects of gender discrimination and reported stress on drug use among racially/ ethnically diverse women in Northern California. Womens Health Issues 2010; 20:211-8.

43. Tran AG, Lee RM, Burgess DJ. Perceived discrimination and substance use in Hispanic/Latino, African-born Black, and Southeast Asian immigrants. Cultur Divers Ethnic Minor Psychol 2010; 16:226-36

44. Whitbeck LB, Hoyt DR, McMorris BJ, Chen X, Stubben JD. Perceived discrimination and early substance abuse among American Indian children. J Health Soc Behav 2001; 42:405-24.

45. Wong CF, Weiss G, Ayala G, Kipke MD. Harassment, discrimination, violence, and illicit drug use among young men who have sex with men. AIDS Educ Prev 2010; 22:286-98.

46. Yoo HC, Gee GC, Lowthrop CK, Robertson J. Selfreported racial discrimination and substance use among Asian Americans in Arizona. J Immigr Minor Health 2010; 12:683-90.

47. Young M, Stuber J, Ahern J, Galea S. Interpersonal discrimination and the health of illicit drug users. Am J Drug Alcohol Abuse 2005; 31:371-91.

48. Bennett GG, Wolin KY, Robinson EL, Fowler S, Edwards CL. Perceived racial/ethnic harassment and tobacco use among African American young adults. Am J Public Health 2005; 95:238-40.

49. Carter RT. Racism and psychological and emotional injury: recognizing and assessing race-based traumatic stress. Couns Psychol 2007; 35:13-105.

50. Bastos JL, Celeste RK, Faerstein E, Barros AJ. Racial discrimination and health: a systematic review of scales with a focus on their psychometric properties. Soc Sci Med 2010; 70:1091-9.

51. Seyferth G. A estratégia do branqueamento. Ciência Hoje 1986; 5:54-6.
52. Silva NV. Uma nota sobre "raça social" no Brasil. In: Hasenbalg C, Silva NV, Lima M, organizadores. Cor e estratificação social. Rio de Janeiro: Contracapa; 1999. p. 107-25.

53. Bastos JL, Peres MA, Peres KG, Dumith SC, Gigante DP. Diferenças socioeconômicas entre autoclassificação e heteroclassificação de cor/raça. Rev Saúde Pública 2008; 42:324-34.

54. Telles EE. Racismo à brasileira: uma nova perspectiva sociológica. Rio de Janeiro: Relume Dumará; 2003.

55. Bastos JL, Faerstein E, Celeste RK, Barros AJ. Explicit discrimination and health: development and psychometric properties of an assessment instrument. Rev Saúde Pública 2012; 46:269-78.

56. Phelan JC, Link BG, Dovidio JF. Stigma and prejudice: one animal or two? Soc Sci Med 2008; 67: 358-67.

57. Krieger N, Sidney S. Racial discrimination and blood pressure: the CARDIA Study of young black and white adults. Am J Public Health 1996; 86:1370-8.

58. Krieger N, Smith K, Naishadham D, Hartman C, Barbeau EM. Experiences of discrimination: validity and reliability of a self-report measure for population health research on racism and health. Soc Sci Med 2005; 61:1576-96.

59. Conde E, Gorman DM. Krieger's conceptualization and measurement of discrimination and internalized oppression in studies of adverse health outcomes. GeoJournal 2009; 74:131-42.

60. Landrine $\mathrm{H}$, Klonoff EA. The schedule of racist events: a measure of racial discrimination and a study of its negative physical and mental health consequences. J Black Psychol 1996; 22:25.

61. Couto PF, Goto JB, Bastos JL. Presão arterial e discriminação interpessoal: revisão sistemática de estudos epidemiológicos. Arq Bras Cardiol 2012; 99:956-63.

62. Meyer IH. Prejudice as stress: conceptual and measurement problems. Am J Public Health 2003; 93:262-5.

63. Bastos JL, Faerstein E. Discriminação e saúde: perspectivas e métodos. Rio de Janeiro: Editora Fiocruz; 2012.

Recebido em 08/Dez/2011

Versão final reapresentada em 30/Out/2012

Aprovado em 22/Nov/2012 